\title{
EFECTOS DEL PROCESO DE BOLONIA EN LA REDUCCIÓN DEL ABANDONO DE ESTUDIOS UNIVERSITARIOS: DATOS PARA LA REFLEXIÓN Y PROPUESTAS DE MEJORA
}

\section{EFFECTS OF THE BOLOGNA PROCESS IN REDUCING THE DROPOUT OF UNIVERSITY STUDIES: DATA FOR REFLECTION AND SUGGESTIONS FOR IMPROVEMENT}

\author{
Lidia Cabrera Pérez \\ Universidad de La Laguna
}

Fecha de recepción: 27/11/2014

Fecha de aceptación: 08/01/2015

\section{RESUMEN}

Uno de los grandes retos del sistema universitario actual es reducir los porcentajes de estudiantes que abandonan sus estudios sin finalizar. En los últimos años las universidades han puesto en práctica acciones destinadas a aumentar el rendimiento y la retención, pero no han teniendo las consecuencias deseadas. Nosotros presentamos en este artículo resultados de una experiencia de aplicación de metodologías activas y de seguimiento continuado de las tareas de los estudiantes, asociadas a la convergencia europea, y su repercusión en la mejora del rendimientos y reducción del abandono, así como opiniones del alumnado y el profesorado sobre los efectos de la reforma en la mejora de la enseñanza. Los resultados muestran que con este tipo de metodologías se consigue que los estudiantes alcancen mayor grado de motivación y compromiso con la institución y, en consecuencia, abandonen menos. Esta forma de enseñar no se ha podido aplicar en los estudios de grado porque no se dispone de las condiciones requeridas para ello. Se aportan sugerencias para la mejora, tanto para los estudiantes tradicionales como para los no tradicionales.

Palabras clave: educación superior; abandono universitario; retención de estudiantes; estudiantes resilientes; aprendizaje basado en problemas; aprendizaje en grupo.

\begin{abstract}
One of the great challenges of the current university system is to reduce the percentage of dropout students. In recent years universities have implemented actions to increase performance and retention haven't had the desired impact. We display in this paper results of an experiment of applying active methodologies and ongoing monitoring of student task, related to European convergence, and its impact on improving student performance and reducting dropout, as well as opinions of students and professor about the effects of reform on improving teaching. The results show that with this type of methodology achieve that students are more motivated, committed to the institution and dropout less. This way of teaching could not apply because grades do not have the conditions required. Suggestions for improvement for both traditional and non-traditional students are reported.
\end{abstract}


Efectos del proceso de Bolonia en la reducción del abandono de estudios universitarios: datos para la reflexión y propuestas de mejora

Key words: higher education; dropout students; Students retention; resilience students; problem based learning; learning in group.

\section{INTRODUCCIÓN}

El abandono de los estudios universitarios sin finalizar ha sido un tema de preocupación y atención creciente en los últimos años, tanto en el ámbito nacional como internacional, pues representa un síntoma claro de crisis, y reducirlo constituye uno de los grandes retos de la enseñanza superior. Actualmente constituye una base para la asignación de recursos y otras decisiones administrativas, además de un importante indicador de desempeño y calidad, incluyéndose en todos los programas de verificación de títulos y evaluación de la calidad. En España empieza a emerger con significatividad entre los años 80 y 90 , asociado al acceso a la universidad de un mayor número de estudiantes con perfiles diversos, y a la masificación de las aulas. Los porcentajes medios a finales de los 90 y principios del 2000 giraban alrededor del $20 \%$ y $25 \%$, alcanzándose cifras muy superiores en las carreras técnicas y en los primeros cursos (Cabrera, Bethencourt, Álvarez y González, 2006). En el curso 2013/14 la media estuvo en $26,1 \%$, con un comportamiento similar en todas las ramas de conocimiento; de estos, el $7,1 \%$ fueron cambios de estudios y del resto (19\%) se desconoce si iniciaron otros estudios o abandonaron el sistema (MEC, 2014).

A pesar de los esfuerzos que se han hecho para informar mejor al alumnado de nuevo ingreso, prevenir el fracaso académico con cursos cero e innovaciones docentes, y mejorar la acogida institucional, variables que se han mostrado constantes en la relación e influencia en la decisión de abandonar, las cifras no descienden. Datos ofrecidos por el Gabinete de Análisis y Planificación de la Universidad de La Laguna (ULL) muestran que en el curso 2010/11 abandonaron un promedio de $24 \%$ en humanidades y ciencias sociales y un $22 \%$ en carreras técnicas; en el curso $2011 / 12$ abandonaron un $28 \%$ en humanidades y un $26 \%$ en técnicas; muchas titulaciones pasan del $40 \%$, llegando incluso hasta el $50 \%$. La tendencia indica un aumento en las ramas de ciencias sociales y humanidades y constancia en la rama científico-tecnológica.

Los datos procedentes de informes de investigación, donde se han hecho estudios retrospectivos y de seguimiento del alumnado, muestran cifras similares (Cabrera y col., 2006; Martín y González, 2010), y superiores si aislamos poblaciones específicas como enseñanza a distancia, estudiantes que combinan estudios y trabajo, estudiantes mayores, etc. (Elías Andreu, 2008; Soler, 2013). Estos subgrupos se encuentran con una estructura universitaria poco adaptada a sus 
características y necesidades, circunstancia que les exige prolongar sus estudios o abandonar.

Esto ha hecho que se califique al sistema universitario español de poco productivo, dado que a una entrada masiva de estudiantes le sigue una elevada tasa de deserción. Si analizamos el panorama internacional donde la media de los países de la OCDE es de un $30 \%$, y algunos países como Italia y EEUU llegan a medias del 55\% (OCDE, 2008, 2010, 2014), y hasta de un 45\% en América-latina (Moncada, 2014; Rojas Betancur y González, 2008), parece que la falta de productividad no es del Estado español sino del sistema universitario en su conjunto. Christie, Munro y Fisher (2004), entre otros, indicaron que esta iba a ser una problemática que afectaría tanto a los países más desarrollados como a los menos desarrollados.

Las cifras españolas preocupan sobremanera porque representan las decisiones y comportamientos de estudiantes de grado, que han sido "supuestamente" beneficiarios de una gran reforma educativa de la enseñanza superior, donde se ha primado el cambio de la forma de enseñar $y$, en consecuencia, se han invertido muchos esfuerzos en la sensibilización y formación del profesorado en el uso de metodologías didácticas y recursos tecnológicos innovadores y favorecedores de una enseñanza más práctica, más motivante y de mayor calidad.

De unos países a otros, sin embargo, se vislumbran casuísticas distintas. Mientras que en los países más industrializados los abandonos están más relacionados con las oportunidades del mercado laboral (Schnepf, 2014), en España hay un fuerte componente personal en el perfil de los estudiantes desertores (Bethencourt y col., 2008), y los estudios realizados en Latinoamérica indican falta de integración y compromiso institucional, bajos resultados y dificultades económicas (García de Fanelli, 2014; Tadeo Lozano, 2010). Schnepf (2014), en las conclusiones de su estudio en el que compara 12 países de la Unión Europea, sugiere una relación directa del abandono con el aprendizaje, al observar como en la mayoría de los países son las chicas las que menos abandonan y también las que tienen notas más altas en secundaria. La relación entre nota de acceso y abandono ha sido una variable contrastada y confirmada en el caso español (MEC, 2014). En el mismo estudio de la Unión Europea se confirma además una relación con el nivel económico y educativo de las familias.

La deserción estudiantil constituye en consecuencia un fenómeno general y dual, pues necesita que se identifiquen y esclarezcan las causas genéricas y específicas que lo originan, y que se encuentren soluciones para reducirlo. Afortunadamente, contamos con importante 
Efectos del proceso de Bolonia en la reducción del abandono de estudios universitarios: datos para la reflexión y propuestas de mejora

literatura, aunque no suficiente, que nos habla de una constelación de variables que han mostrado estar relacionadas con la decisión de abandonar, así como sobre la mayor y menor influencia de unas sobre otras (Arriaga, Burillo, Carpeño y Casaravilla, 2011; Cabrera y col. 2006; Elías Andreu, 2008), y con diversidad de propuestas de acciones y programas educativos para fomentar la retención.

En la Tabla 1 presentamos una compilación del conjunto de factores y variables influyentes en el abandono (González, Alvarez, Bethencourt y Cabrera, 2007; Elías, 2008; Inhitani y DesJardins, 2003; Landry, 2003; Latiesa, 1992; Pascarella y Terenzini, 1991; Peterson, 2002; Taylor y Bedford, 2004; Yorke, 2000, entre otros). Hay que tener en cuenta que estas variables no afectan del mismo modo atendiendo al contexto social y la política educativa de cada país, a los distintos tipos de abandono (abandono de la carrera, de la institución, del sistema educativo), o si se trata de un abandono temporal o definitivo; tampoco se comportan del mismo modo los abandonos tempranos, que se producen en primero y segundo de carrera, que los abandonos tardíos. Arriaga y col., (2011) encontraron que los estudiantes que cambian de titulación y siguen estudiando en la misma universidad son los que muestran mayor implicación académica y mayor resistencia a la presión, mientras que los que abandonan definitivamente muestran muy baja valoración de la utilidad de los estudios, a pesar de que la imagen que tienen de la universidad no es mala.

En cualquiera de los casos, tal y como apuntó Tinto (1993), debe tenerse en cuenta que cada historia de abandono se va construyendo lentamente, y de un modo singular. Por tanto, si queremos reducir el problema de la deserción tenemos que actuar más sobre los casos que sobre los grupos, realizando investigaciones más cualitativas y biográficas y haciendo intervenciones educativas más personalizadas sobre los casos riesgo.

\begin{tabular}{|l|l|l|}
\hline PSICOLÓGICAS & Motivación & $\begin{array}{l}\text { Motivación y decepción carrera } \\
\text { Expectativas futuras }\end{array}$ \\
\cline { 2 - 3 } & Persistencia & $\begin{array}{l}\text { Capacidad de esfuerzo } \\
\text { Demorar recompensas } \\
\text { Tolerancia a la frustración }\end{array}$ \\
\hline EDUCATIVAS & Elección de estudios & $\begin{array}{l}\text { Realizar estudios elegidos } \\
\text { Visión realista de la carrera }\end{array}$ \\
\cline { 2 - 3 } & Capacidades & $\begin{array}{l}\text { Nota de acceso } \\
\text { Conocimientos previos } \\
\text { Dificultades para aprobar } \\
\text { Rendimiento académico }\end{array}$ \\
\cline { 2 - 3 } & Compromiso & $\begin{array}{l}\text { Dedicación al estudio } \\
\text { Asistencia a clase }\end{array}$ \\
\cline { 2 - 3 } & Estrategias de estudios & $\begin{array}{l}\text { Constancia y disciplina } \\
\text { Método de estudio }\end{array}$ \\
\hline
\end{tabular}




\begin{tabular}{|c|c|c|}
\hline \multirow[t]{2}{*}{ EVOLUTIVAS } & Inmadurez & $\begin{array}{l}\text { Académica } \\
\text { Personal }\end{array}$ \\
\hline & Inadaptación & $\begin{array}{l}\text { Percepción de poco apoyo } \\
\text { institucional } \\
\text { Percepción de poca ayuda } \\
\text { Ausencia de vínculos grupales }\end{array}$ \\
\hline \multirow[t]{2}{*}{$\begin{array}{l}\text { CIRCUNSTANCIAS } \\
\text { VITALES }\end{array}$} & Sobre la carrera & $\begin{array}{l}\text { Incompatibilidad trabajo y estudio } \\
\text { Falta de tiempo } \\
\text { Compromiso institucional }\end{array}$ \\
\hline & Ajenas a la carrera & $\begin{array}{l}\text { Enfermedades } \\
\text { Cargas familiares } \\
\text { Otras dificultades }\end{array}$ \\
\hline FAMILIARES & Influencia elección & $\begin{array}{l}\text { Elección de estudios } \\
\text { Presión por las calificaciones }\end{array}$ \\
\hline ECONÓMICAS & $\begin{array}{l}\text { Universidad } \quad \text { Pública } \\
\text { /Privada }\end{array}$ & $\begin{array}{l}\text { Deseo de tener dinero e independizarse } \\
\text { Dificultades pago matrículas }\end{array}$ \\
\hline \multirow[t]{4}{*}{ INSTITUCIONALES } & $\begin{array}{l}\text { Información } \\
\text { orientación para } \\
\text { acceso }\end{array}$ & $\begin{array}{l}\text { Orientación vocacional } \\
\text { Inadaptación a las necesidades de los } \\
\text { nuevos estudiantes (perfiles educativos, } \\
\text { preferencias profesionales, culturas) }\end{array}$ \\
\hline & Integración institucional & $\begin{array}{l}\text { Satisfacción con el ambiente aula y } \\
\text { centro } \\
\text { Relación entre los estudiantes } \\
\text { Apoyo ante dificultades } \\
\text { Compromiso con la institución }\end{array}$ \\
\hline & Plan estudio & $\begin{array}{l}\text { Conexión mundo laboral } \\
\text { Muy teórico } \\
\text { Clases poco dinámicas } \\
\text { Excesiva exigencia } \\
\text { Poco adaptado a los estudiantes que } \\
\text { trabajan }\end{array}$ \\
\hline & Profesorado & $\begin{array}{l}\text { Poco motivador } \\
\text { Poca atención personalizada } \\
\text { Relación con los estudiantes } \\
\text { Masificación: no aplicar metodologías } \\
\text { activas }\end{array}$ \\
\hline \multirow[t]{2}{*}{ SOCIALES } & Salidas profesionales & $\begin{array}{l}\text { Orientación profesional } \\
\text { Oferta laboral cualificada/ajustada } \\
\text { titulación } \\
\text { Alejamiento entre empresa y } \\
\text { universidad }\end{array}$ \\
\hline & Valor social de estudios & $\begin{array}{l}\text { Poca valoración social de estudios } \\
\text { universitarios } \\
\text { Percepción que la universidad no les } \\
\text { pertenece }\end{array}$ \\
\hline
\end{tabular}

Tabla 1. Factores y variables relacionadas con el abandono.

Las variables más influyentes son las dificultades del alumnado para superar las materias y la desmotivación, por falta de ayuda o porque los estudios no responden a sus expectativas. Los estudios comparativos entre unos estudiantes y otros nos muestran que los que persisten suelen ser más sistemáticos en el trabajo diario y se esfuerzan 
Efectos del proceso de Bolonia en la reducción del abandono de estudios universitarios: datos para la reflexión y propuestas de mejora

más. Por el contrario, los que abandonan no poseen las estrategias necesarias para superar obstáculos y ser persistentes en sus metas.

Nosotros, en estudios realizados con estudiantes de la Universidad de La Laguna (Bethencourt, 2008; González y col., 2007), encontramos que lo que más diferencia a unos y otros es que los estudiantes que continúan asisten regularmente a clase, preguntan dudas al profesor y llevan las tareas al día, persisten para acabar la carrera a pesar de los obstáculos (con un efecto muy grande), están más satisfechos y motivados con la carrera, tienen mayor capacidad para demorar las recompensas, y existe en ellos un mayor ajuste entre sus capacidades y las exigencias de la titulación. Arriaga y col. (2011), en otro estudio comparativo con estudiantes de la Universidad Politécnica de Madrid, encuentran que los que se quedan se esfuerzan y trabajan más, tienen una visión más realista de la carrera y sienten que la institución si les ha ayudado a superar las dificultades.

Los estudios sobre resiliencia también constatan la gran relevancia de las características del alumnado resiliente o persistente en la evitación del abandono. En este sentido, no se trataría tanto de las causas que provocan el abandono, sino de los mecanismos que los estudiantes ponen en marcha para afrontar las dificultades y obtener sus metas. El perfil de este alumnado se ha definido como aquel que tiene: competencia social, autocontrol, autonomía, responsabilidad, inteligencia, destrezas en la solución de problemas, pensamiento crítico, iniciativa, aspiraciones educativas, propósito en sus vidas, esperanza, optimismo, perseverancia, espiritualidad, sentido del humor, motivación y autoestima (Bethencourt y col, 2008). Por el contrario, los estudiantes que no son resilientes y abandonan la universidad serían aquellos que, según Bernard (1996), no son autoeficaces, tienen baja autoestima, poseen pocas habilidades cognitivas, no son perseverantes, y fueron poco reforzados en su motivación, autoestima y destrezas de afrontamiento.

Estos estudios también han encontrado que los estudiantes son más resilientes cuando conviven y aprenden en instituciones favorecedoras de resiliencia, que propician ambientes afectivos, en condiciones de trabajo placenteras, en las que los profesores manejan bien los grupos, tienen altas expectativas sobre sus estudiantes y fomentan la motivación intrínseca (Wang, Haertel y walberg, 1999).

En los últimos años están emergiendo factores económicos y sociales relacionados con la decisión de abandonar, como la escasa oferta laboral (Reques, 2007), o las dificultades para compatibilizar estudios y trabajo (Soler, 2014). Elías Andreu (2008), en un estudio 
realizado en la Universidad autónoma de Barcelona, encontró que el $68 \%$ de los estudiantes que abandonaron también trabajaba, y el $25 \%$ manifestó que dejaron de estudiar porque no podían combinar ambas cosas, y porque creían que los estudios que estaban realizando no les iban a facilitar mejores oportunidades laborales.

La reforma experimentada por las universidades con el Plan Bolonia para la convergencia europea ha sido una gran oportunidad para la mejora, ya que con ella se han introducido planes de estudio más vinculados con el mundo laboral, metodologías docentes más activas, recursos didácticos más tecnológicos, y modalidades de enseñanza distintas a las clases teóricas y prácticas tradicionales, factores que se revelan efectivos en la aminoración de los porcentajes de abandono.

Todos los estudios sobre aprendizajes basados en problemas (ABP) y las prácticas docentes centradas en el alumnado mejoran la calidad de los aprendizajes y la integración y compromiso institucional, pues los aprendizajes adquiridos van más allá del conocimiento, incluyendo estrategias cognitivas más elevadas de aplicación y análisis. Además, los estudiantes se motivan tratando de solucionar los problemas, lográndose con ello una alta participación e implicación en el estudio, y una mejor preparación para resolver problemas del mundo laboral y social. Estas y otras evidencia ya señaladas hicieron plantearnos si los grandes esfuerzos realizados con la convergencia europea están surtiendo efecto en la calidad de la enseñanza y en los resultados educativos, sobre todo en la mejora del rendimiento y la disminución del abandono.

\section{MÉTODO}

En el estudio que aquí presentamos, nosotros quisimos comprobar si los cambios metodológicos sobre el proceso de enseñanza y aprendizaje promovido con el proceso de convergencia europea estaban teniendo repercusiones en las tasas de rendimiento y abandono.

Para dar respuesta a estas cuestiones presentamos resultados sintetizados de dos estudios: resultados de una experiencia de aplicación de metodologías docentes activas y participativas y su influencia en el rendimiento', y resultados de un estudio de opinión del

\footnotetext{
1 Esta experiencia fue promovida y subvencionada por el Vicerrectorado de calidad y nuevos estudios de la Universidad de La Laguna. Los datos son los resultantes de la evaluación realizada por Lidia Cabrera.
} 
Efectos del proceso de Bolonia en la reducción del abandono de estudios universitarios: datos para la reflexión y propuestas de mejora

alumnado y profesorado sobre la mejora del rendimiento y la calidad en las enseñanzas en los estudios de grado? participativas

a) Estudio de Experimentación con metodologías activas y

En este estudio se desarrollaron 20 proyectos de experimentación con las metodologías alternativas, en los que se trató de incluir más competencias procedimentales y actividades prácticas, métodos de enseñanza demostrativos, atención más personalizadas y seguimiento del trabajo el alumnado, modalidades de evaluación continua y pruebas cualitativas, mayor uso de tecnologías de la información y enseñanza on-line.

En estos proyectos participaron 276 profesoras y profesores, de las titulaciones de Bellas Artes, Derecho, Empresariales, Filología Hispánica, Filología Inglesa, Filosofía, Historia, Historia del Arte, Ingeniería Informática, Ingeniería Química, Ingeniería Técnica Industrial, Ingeniería Técnica en Obras Públicas, Logopedia, Maestro en Educación Primaria, Maestro en Lengua Extranjera, Matemáticas, Pedagogía, Química, Trabajo Social, y Turismo.

Por cada proyecto se elaboró una memoria que incluía procedimiento, resultados obtenidos, dificultades y sugerencias de mejora. Estas memorias fueron analizadas atendiendo a un sistema de categorías e indicadores.

b) Estudio de opinión del profesorado y alumnado sobre la calidad de la enseñanza en los estudios de grado

La muestra estuvo integrada por 507 estudiantes voluntarios de grado de la Universidad de La Laguna, de las titulaciones de Maestro, Pedagogía, Psicología, Logopedia, Trabajo Social y Fisioterapia, y 24 de sus profesores. 269 estudiantes cursaban estudios de primero y 238 de segundo. Sus edades oscilaban entre 18 y 53 años, si bien el grupo mayoritario tenía 18 y 19 años, 400 mujeres y 107 hombres. Los profesores tenías entre 37 y 69 años, 13 hombres y 11 mujeres.

Para la recogida de datos se diseñaron dos cuestionarios, uno para el alumnado y otro para el profesorado, que se administraron de forma presencial dentro y fuera del aula.

2 Los datos de este estudio fueron recogidos por la becaria de colaboración del MEC Yanna Mufreger; también se contó con la colaboración del profesor José Tomás Bethencourt. 


\section{RESULTADOS}

\subsection{Resultados sobre desarrollo de metodologías activas y sus efectos en el rendimiento}

El trabajo docente llevado a cabo en estas experiencias metodológicas requirió de una reorganización de la enseñanza que se caracterizó por ser: más activa, al fomentarse la realización de ejercicios y tareas prácticas; más práctica, de demostración y aplicabilidad de los conocimientos aprendidos; de seguimiento del trabajo del alumnado en tutorías académicas, para lo que se requirió agrupar a los estudiantes en grupos entre 3 y 10 personas.

La valoración del profesorado implicado sobre los resultados obtenidos fue muy positiva, pues, según ellos, supuso un avance en la mejora de la docencia, puesto que pasaron de una posición de "enseñar" a "enseñar a aprender", y en la mejora del rendimiento y madurez de los estudiantes. Para ellos, la experimentación en sí misma ya ha supuesto un cambio, pues les ha llevado a asumir e interiorizar un nuevo modelo, y a tener otra disposición que incidirá en sus prácticas futuras, a pesar de las limitaciones encontradas. A continuación mostramos los aspectos más destacados.

a) Mayor número de horas de trabajo requeridas para el alumnado y el profesorado

Esta modalidad docente exige al alumnado trabajar de forma continuada e invertir más horas que las requeridas en el modelo tradicional para llevar las tareas al día. El $80 \%$ del alumnado se queja del número de horas diarias que tiene que dedicar, aunque también reconoce que aprenden y aprueban más, y que el esfuerzo realizado a lo largo del curso se ve compensado al final, al ver que no tienen que estudiar tanto para los exámenes. El profesorado opina que el tiempo invertido por el alumnado es diverso, dependiendo del nivel previo y madurez de cada uno, y que sólo los estudiantes con niveles más bajos tuvieron que dedicar muchas horas al trabajo personal. Un 30\% considera que el alumnado no dedica el tiempo suficiente al estudio, y que los que señalan que el trabajo es excesivo son los que carecen de hábito de estudio y planificación para la realización de tareas. Un 20\% manifestó que muchos estudiantes siguen dejando el desarrollo de las tareas para el final, y que en estos casos no se consiguieron mejoras significativas. A pesar de ello, el profesorado considera que este método es idóneo para aprovechar mejor las horas de clase, conseguir más objetivos y fomentar hábitos de trabajo y la motivación de logro en el alumnado. 
Efectos del proceso de Bolonia en la reducción del abandono de estudios universitarios: datos para la reflexión y propuestas de mejora

La mayor dificultad de este modelo reside en la dedicación docente que requiere, sobre todo cuando los grupos son numerosos. Fundamentalmente se necesita de más tiempo para la preparación de materiales, la corrección de actividades con inmediatez, las tutorías de seguimiento en pequeños grupos, la coordinación entre materias y la docencia virtual, que exige un mayor número de horas. El profesorado estima que un esfuerzo y dedicación de estas características es posible en modo experimental, pero no de un modo continuado, a menos que la dedicación docente se desarrolle con grupos más pequeños. Generalizar esta metodología de trabajo al total de asignaturas y con el número de estudiantes actuales, sobrepasaría las posibilidades reales de dedicación de cualquier profesor y dificultaría el óptimo desarrollo de otras actividades profesionales como las de investigación.

b) Logro metodológico y de éxito académico de las tutorías académicas

Las tutorías académicas con los pequeños grupos se llevaron a cabo cada 15 días aproximadamente. En ellas se revisaron los trabajos individuales y grupales realizados previa demanda, se hicieron exposiciones individuales y grupales y se resolvieron ejercicios de entrenamiento para la evaluación.

Esta actividad fue muy valorada, tanto por el alumnado como por el profesorado, pues según ellos es donde radica el alcance del cambio metodológico, ya que permite: el aprendizaje de competencias prácticas a través de ejercicios de aplicación, detectar dificultades del alumnado, corregir errores a partir del feedback mostrado y motivar al alumnado en el estudio y trabajo continuado. El alumnado manifestó que el feedback recibido fue lo que determinó el éxito y que en las tutorías aumentaban su motivación por la carrera, al ver la utilidad de los aprendizajes para la solución de problemas prácticos.

\section{c) La evaluación continua pieza clave para mejorar el rendimiento}

Asociada a la tutoría académica se desarrolló la evaluación continua, fundamentalmente con pruebas de carácter cualitativo. Para el $100 \%$ del profesorado esta modalidad de evaluación fue otra de las piezas clave y diferenciadora respecto de los modelos anteriores, y por la que se obtuvieron mejoras en el rendimiento de los estudiantes. Los trabajos prácticos permitían valorar si los estudiantes estaban consiguiendo las competencias previstas; además se comprobó que con ellos aprendían más y mejor. 
d) Repercusiones en el Rendimiento académico y disminución del abandono

En general, se observó una mejora en el rendimiento de los estudiantes y un incremento del número de presentados. Sin embargo, los porcentajes de aprobados dependieron del carácter de la asignatura (obligatoria/optativa) y de la titulación cursada. En las asignaturas optativas, donde el número de alumnado era más reducido y el procedimiento de evaluación continuo en su totalidad, los aprobados ascendieron hasta un $90 \%$ y $100 \%$. En el resto de asignaturas se observó también una disminución del número de no presentados, y los índices de suspensos se redujeron de manera que pasaron de medias del $35 \%$ en cursos anteriores al $17 \%$. El profesorado de titulaciones técnicas insiste en informar sobre el escaso nivel de conocimientos previos del alumnado y su repercusión en los suspensos.

Gran parte del alumnado se comprometió con la modalidad de evaluación continua, pero luego abandonaron para presentarse al examen final (convocatoria oficial), porque les pareció que ello exigía menos dedicación; en ellos no se observaron cambios en el rendimiento, y sí mucha apatía hacia el aprendizaje. El profesorado concluye que los estudiantes que asisten con regularidad a clase, participan en las actividades diarias y trabajan de manera continuada superan la asignatura en la primera convocatoria.

\section{e) Características del alumnado}

Las características del alumnado constituyeron una dificultad y un reto metodológico, pues los profesores observaron que con esta metodología los estudiantes lograron desarrollar importantes habilidades para el trabajo intelectual y de equipo, a pesar de que hubo que dedicar mucho tiempo a enseñarles, y que había estudiantes que no se comprometían con el grupo. Las mayores limitaciones encontradas en los estudiantes fueron las siguientes: falta de competencias instrumentales para la realización de este tipo de actividades (búsqueda de información, realización de memorias, evitar el plagio, etc.), carencia de competencias básicas como conocimientos previos de la materia, limitaciones lingüísticas, escaso nivel de inglés, escaso nivel cultural, falta de autonomía y necesidad de mucho apoyo externo. 
Efectos del proceso de Bolonia en la reducción del abandono de estudios universitarios: datos para la reflexión y propuestas de mejora

f) Propuestas para lograr un cambio de actitudes y la mejora de competencias y habilidades del alumnado

A partir de esta experiencia, el profesorado considera que, para que el proceso de reforma cumpla con sus finalidades y se alcancen mejores resultados, la universidad debería introducir las siguientes mejoras:

1. En la organización docente: incrementar las horas prácticas de las materias e incluir horas obligatorias de trabajo tutelado, disminuir los horarios de clase para que el alumnado tenga tiempo para asistir a tutorías y hacer trabajos de investigación, aumentar la coordinación entre materias para distribuir mejor el volumen de trabajo del alumnado, reducir el número de alumnado por grupo y posibilitar la realización de más salidas de campo.

2. En el profesorado: aumentar la plantilla del profesorado con objeto de favorecer el desdoblamiento de grupos, contabilizar en el plan docente del profesorado las horas de trabajo tutelado con el alumnado, fomentar el uso de plataformas virtuales y nuevas tecnologías y favorecer la comunicación de experiencias de estas características entre el profesorado.

3. Infraestructuras: acondicionar espacios físicos, sobre todo, en lo que se refiere a dotación de seminarios y modernización de los laboratorios, mejorar los espacios de las aulas y sus equipos informáticos y audiovisuales y mejorar las estructuras administrativas de las facultades.

4. Alumnado: ofertar cursos de formación en competencias básicas y conocimientos previos (los cursos cero son insuficientes), poner en práctica estrategias metodológicas docentes que exijan al alumnado más dedicación al trabajo personal, formación en estrategias y habilidades para la realización de trabajos académicos que requieran análisis y razonamiento y fomentar su participación en el aula (Ver Tabla 2).

\begin{tabular}{|l|l|l|l|}
\hline $\begin{array}{l}\text { Deficiencias del alumnado para } \\
\text { este tipo de enseñanza }\end{array}$ & $\%$ & $\begin{array}{l}\text { Cambios observados en el } \\
\text { alumnado tras la experiencia }\end{array}$ & $\%$ \\
\hline $\begin{array}{l}\text { Poco participativo, poco } \\
\text { autónomo, no toma decisiones, } \\
\text { espera que le indiquen lo que } \\
\text { tiene que hacer }\end{array}$ & $46 \%$ & $\begin{array}{l}\text { Mayor implicación en el proceso } \\
\text { de aprendizaje (consultas } \\
\text { bibliográficas, más activos, } \\
\text { asistencia tutorías, etc.) }\end{array}$ & $22 \%$ \\
\hline $\begin{array}{l}\text { Escasas competencias lingüísticas } \\
\text { (oral y escrita) }\end{array}$ & $11^{\prime} 5 \%$ & $\begin{array}{l}\text { Actitud más positiva para } \\
\text { aprender ymayor interés }\end{array}$ & $333^{\prime} 3 \%$ \\
\hline Pocos conocimientos previos de & $30^{\prime} 7 \%$ & \begin{tabular}{l} 
Mejor actitud para trabajar en \\
\hline
\end{tabular}
\end{tabular}




\begin{tabular}{|l|l|l|l|l|}
\hline $\begin{array}{l}\text { la materia, poca madurez para } \\
\text { estudiar (solo copia), pocas } \\
\text { habilidades de organización del } \\
\text { estudio }\end{array}$ & $\begin{array}{l}\text { grupo y aprender de los } \\
\text { compañeros }\end{array}$ & \\
\hline $\begin{array}{l}\text { Poca dedicación al estudio } \\
\text { (tiempo) }\end{array}$ & $7^{\prime} 6 \%$ & $\begin{array}{l}\text { Asimilación más profunda de } \\
\text { aprendizajes }\end{array}$ & $1^{\prime} 1 \%$ \\
\hline $\begin{array}{l}\text { Escaso compromiso con los } \\
\text { estudios y su futuro profesional }\end{array}$ & $33^{\prime} 7 \%$ & $\begin{array}{l}\text { Mayor cumplimiento de los plazos } \\
\text { para entregar trabajos y ejercicios }\end{array}$ & $11^{\prime} 1 \%$ \\
\hline Poca motivación & $3^{\prime} 7 \%$ & \multicolumn{2}{l}{} \\
\hline
\end{tabular}

Tabla 2. Opiniones del profesorado sobre cambios observados y requeridos en el alumnado.

\subsection{Resultados de opinión del alumnado y profesorado sobre los estudios de grado}

\subsubsection{Opiniones del alumnado}

a) Elección y acceso a estudios

El $64 \%$ de los estudiantes eligió los estudios que está cursando en primera opción y el resto no está realizando los estudios elegidos. Los motivos que tuvieron en cuenta para elegir esos estudios fueron mayoritariamente vocacionales; otras variables como influencia de amigos y familiares, la nota de acceso, la información sobre la carrera, el prestigio y salida profesional que tienen los estudios tuvieron poca influencia en sus decisiones.

b) Clima académico y contenido de los estudios

El clima académico en el que se desarrollaron los estudios se percibe en general de manera positiva. Alrededor del $75 \%$ siente que las relaciones del grupo con el profesorado y entre el alumnado son cordiales o bastante cordiales. Sin embargo, se observa que de primero a segundo estas apreciaciones se van volviendo más negativas. En las relaciones con el profesorado, un $58 \%$ de los de primero y un $43,6 \%$ de los de segundo se sitúan en el extremo positivo de la escala (puntuaciones de 8,9 y 10). En las relaciones entre ellos, en primero se sitúan en el extremo positivo de la escala (puntuaciones de 8,9 y 10) un $44,7 \%$, y solo un $26,1 \%$ en segundo.

Las valoraciones que los estudiantes hacen acerca de la preparación y cumplimiento del profesorado son altamente positivas; concretamente, el $96 \%$ de los estudiantes de primero y el $81,5 \%$ de los de segundo consideran que la calidad formativa del profesorado es 
Efectos del proceso de Bolonia en la reducción del abandono de estudios universitarios: datos para la reflexión y propuestas de mejora

buena. Sin embargo, además del descenso de porcentajes en las valoraciones positivas globales, observamos que frente al $68,8 \%$ de los estudiantes de primer curso que los califican como excelentes (puntuaciones de 9 y 10 de la escala), en segundo solo muestran esa valoración $14,2 \%$, descendiendo las puntuaciones a 8,7 y 6 . Ambos grupos consideran que se fomenta el trabajo grupal y el aprendizaje autónomo, en un porcentaje bastante alto.

La mayoría de los estudiantes de primero consideran que los contenidos de las asignaturas se ajustan al plan de estudios, si bien con niveles de ajuste distinto. Por su parte, el 55,8\% de los de segundo considera que los contenidos no están relacionados con el plan de estudios. La valoración que hacen sobre las aulas virtuales vuelve a ser desigual; mientras los de primero consideran en su mayoría que el uso de las aulas virtuales les ayuda a trabajar mejor las materias, haciéndolas más asequibles y obtener mejores aprendizajes y mayor rendimiento en la carrera, el $31,9 \%$ de segundo cree que el uso del aula virtual no influye en el rendimiento, y el $50 \%$ dice que no facilita el aprendizaje. Debemos señalar que alrededor del $90 \%$ de ambos grupos asiste regularmente a clase.

En relación con las percepciones sobre si la carrera les está dando una buena formación para el desarrollo profesional y el futuro laboral, vuelve a haber un descenso importante en las valoraciones de primero a segundo; un $80 \%$ de primero y un $59,8 \%$ de segundo lo valoran positivamente. En cualquier caso, los estudiantes sienten que la formación universitaria no es suficiente y que tendrán que completarla por otras vías. También es importante destacar que en ambos grupos las valoraciones son positivas, pero con posiciones medias. Únicamente el $21,5 \%$ en primero y el $11 \%$ en segundo opinan que alcanzan una alta preparación en la titulación.

\section{c) Rendimiento académico}

En lo que se refiere a la percepción que los estudiantes tienen acerca de su capacitación para cursar los estudios, hemos de señalar que el $85 \%$ se siente muy capacitado para hacer la carrera y le otorga mucho valor a las capacidades personales, tanto para elegir como para realizar la carrera.

En relación con el rendimiento, el $29,8 \%$ de los estudiantes de primero y el $36,1 \%$ de los de segundo dicen haber tenido un rendimiento alto; el $55,8 \%$ de primero y el $53,4 \%$ de segundo dicen que su rendimiento fue medio; el $14,5 \%$ en primero y el $10,1 \%$ en segundo señalaron que tuvieron un rendimiento bajo. 
d) Satisfacción con los estudios

Un $66,2 \%$ en primero y un $52,9 \%$ en segundo se encuentran altamente motivados con los estudios que están realizando; el $29 \%$ de los estudiantes de primero y el 39,9 de los de segundo tienen una motivación media; finalmente, el $4,8 \%$ de los de primero y el $9,2 \%$ de segundo no se sienten motivados. De nuevo, observamos como de un curso a otro el alumnado se va desencantando con los estudios.

Por lo que concierne a sus intenciones y perspectivas de futuro, llamó mucho la atención el hecho de que el $12 \%$ de estudiantes de primero y el $3,8 \%$ de segundo tuviesen claras intenciones de dejar los estudios que están cursando. También debe destacarse el que un 12\% en primero y un $19,6 \%$ en segundo presenten considerables dudas puesto que ello supone un claro indicio de abandono. Las razones pueden ser diversas, pero los altos índices de insatisfacción con la carrera justifican suficientemente la decisión. Los niveles de satisfacción son los siguientes: solo el $48 \%$ en primero y el $30,7 \%$ en segundo se sienten muy satisfechos; el resto muestra dentro de la escala valores en descenso, situándose entre "medianamente satisfecho" y "poco satisfecho".

\subsubsection{Opiniones del profesorado}

Las valoraciones del profesorado sobre el proceso de Bolonia y la situación actual de la enseñanza se sintetizan en las consideraciones siguientes:

1. El $100 \%$ del profesorado piensa que realizar los estudios elegidos en primera opción es un determinante importante de éxito, con un nivel de influencia alto o muy alto.

2. El $75 \%$ ha recibido formación para la introducción de tecnologías de la información y comunicación en el aula, y las ponen en práctica (87\%); sin embargo, considera y opina que la utilización de plataformas de docencia on-line no está relacionada con la mejora del rendimiento $(39,1 \%)$, y que tampoco lo está la utilización de otros recursos TIC. Actualmente, el $87 \%$ manifiesta que asiste regularmente a cursos y actividades formativas para la mejora de la docencia.

3. El clima creado en el aula ayuda mucho a aumentar el aprendizaje y la convivencia (100\%).

4. Para el $45,8 \%$ la reforma de los títulos no ha supuesto ningún cambio ni ha repercutido en las metodologías de enseñanza que emplea; para el resto, $54,2 \%$, sí ha influido. 
Efectos del proceso de Bolonia en la reducción del abandono de estudios universitarios: datos para la reflexión y propuestas de mejora

5. El $54 \%$ considera que los cambios que teóricamente se han introducido para la mejora de la calidad y el aumento del rendimiento de los estudiantes no han visto su plasmación práctica; el resto considera que sí. El $75 \%$ está poco satisfecho con el modo en el que se ha introducido todo el proceso de Bolonia, y considera que debería ser sustituido por otro modelo educativo.

\section{CONCLUSIONES}

Los datos analizados parecen indicar que el abandono universitario, más que un problema, es connatural a la educación superior, por lo que no sería disparatado plantear si las estrategias de las universidades tienen que dirigirse a reducirlo o a gestionarlo, adaptando sus estructuras y sus enseñanzas a este nuevo alumnado, que interrumpe sus estudios, se va a otra universidad, vuelve, simultanea estudios y trabajo, accede a la universidad después de los 25 años y termina su carrera a lo largo de su vida sin ajustarse a la periodicidad diseñada en los planes de estudio. Las estadísticas de los informes educativos anuales de la OCDE muestran que, aunque no se titulen, estos estudiantes que abandonan encuentran más fácilmente empleo, obtienen mejores notas si realizan estudios secundarios especializados, y un $40 \%$ de ellos adquiere títulos superiores en momentos posteriores de sus vidas (OCDE, 2014). Por tanto, siguiendo la teoría del capital humano, solo haber ido a la universidad se convierte en una ganancia para ellos y para el mercado laboral.

La realidad española actual, sin embargo, refleja más a un alumnado intelectualmente inmaduro, que ha iniciado estudios inadecuados, por desconocimiento o porque no obtuvo plaza en los que deseaba, y que además no posee las estrategias necesarias para afrontar dificultades, que a un alumnado que construye sus decisiones vocacionales y que se va formando simultaneando estudios y trabajo, como consecuencia de una realidad económica y social inestable y cambiante. En este estudio, tras el análisis de la literatura y de los resultados de la aplicación de metodologías de trabajo práctico y continuado asociadas a la reforma para la convergencia europea, confirmamos, una vez más, que las características que definen el perfil de estudiantes no comprometidos con los estudios y desertores son: tienen una percepción ideal de la carrera antes de entrar y poseen peores notas de acceso, no llevan las tareas al día, no están motivados con la carrera, sus capacidades no están ajustadas a las exigencias de la carrera, carecen de habilidades y madurez para el trabajo intelectual, se esfuerzan menos y sienten que la institución y los profesores no les ayudan lo suficiente. 
Pero atribuir y delegar toda la responsabilidad al alumnado sería una interpretación muy simplista, y de alusión de responsabilidades por parte de la institución, pues ni el alumnado es el único responsable de ser como es, ni el único afectado. El abandono también afecta a las familias, a las instituciones universitarias (con importantes efectos académicos de baja calidad institucional y poca responsabilidad social) y a la sociedad (con efectos financieros y de perdida de recursos, ya que ve disminuido su capital social de la tasa de graduados). Además, en la decisión de abandonar confluyen diversos factores que exigen que se compartan responsabilidades, como la formación previa, los códigos y experiencias culturales de procedencia, el acceso y la integración en la institución, dificultades en la actividad académica como consecuencia de la masificación, la poca vinculación con los docentes y la escasez de salidas laborales.

Nos encontramos entonces ante dos situaciones que requieren ser atendidas, las de los estudiantes tradicionales que tienen dificultades para adaptarse a la institución, y la de los estudiantes no tradicionales para los que la universidad no tiene planes alternativos. En consecuencia, se requiere de acciones que compensen las carencias de los primeros y de otras modalidades formativas para los segundos.

Las universidades son actualmente las únicas empoderadas para desarrollar enseñanzas más adaptadas a los distintos perfiles de estudiantes y a sus necesidades, y para crear ambientes de aprendizajes resilientes. Además, tienen la responsabilidad social de rescatar al mayor número de alumnado posible e incentivarle para que no abandone. A pesar de que la escasa oferta laboral se ha mostrado como la principal fuente de desmotivación de los estudiantes, los informes de la OCDE también muestran que las tasas de paro son inferiores en los titulados universitarios, aún en tiempos de crisis (de un $15,2 \%$ frente al $25 \%$ del resto de población), por lo que la educación constituye un seguro frente al desempleo, a pesar de que en España en los primeros años realizan trabajos para los que no se requiere cualificación universitaria, y los ingresos no mejoran por tener más titulación, en contra de lo que sucede en otros países (OCDE, 2014).

Hasta ahora han sido muchas las iniciativas desarrolladas en la lucha contra la deserción, pero estas no están teniendo todo el éxito pretendido, no solo porque no se adaptan a todos los tipos de abandono, sino porque las universidades no disponen de las infraestructuras necesarias para aumentar su eficacia, como la disminución del número de estudiantes por curso, condición necesaria para desarrollar el modelo de enseñanza eficaz que llevaba implícito el Plan Bolonia y que se ha presentado en este trabajo. 
Efectos del proceso de Bolonia en la reducción del abandono de estudios universitarios: datos para la reflexión y propuestas de mejora

En los estudios mostrados el profesorado valora muy positivamente el cambio metodológico introducido con la convergencia europea como factor determinante para la mejora del aprendizaje, el rendimiento, la motivación hacia los estudios y la persistencia, no obstante considera que no está teniendo efectos porque no se ha contado con los recursos suficientes, ni se ha cambiado en profundidad la estructura de enseñanza. A conclusiones similares han llegado Daza y Elías (2013) y Elias (2008), quienes afirman que la reforma será efectiva en la retención de estudiantes cuando se tengan otras condiciones de aprendizaje. Morales, Pardo y Álvarez (2010) valoran la oportunidad de la reforma y sus efectos positivos sobre el aprendizaje y la reducción del abandono, pero igualmente poniendo como requisito las condiciones de aprendizaje, es decir, espacios y horarios adaptados al trabajo con pequeños grupos, tutorías de seguimiento de aprendizajes, sistemas flexibles para el estudio, mejor formación del profesorado, etc.

Otras conclusiones del profesorado fueron que las nuevas tecnologías y la enseñanza on-line no son en sí mismas herramientas de mejora, mientras que si lo son el clima de convivencia en el aula, el seguimiento de trabajos, los aprendizajes compartidos, etc. y que esto es posible conseguirlo solo con el trabajo en pequeños grupos.

De la opinión de los estudiantes se desprende como un modelo de trabajo tradicional, masivo y competitivo les va cambiando sus percepciones sobre la universidad, la carrera y el clima del aula, y empiezan a valorar las salidas laborales; si no ven posibilidades inmediatas sus nivel de satisfacción van cambiando y la motivación por los estudios disminuyendo. Sin embargo, cuando se desarrollaron los planes docentes experimentales, donde se atendieron estas necesidades y dificultades, los efectos sobre la motivación de los estudiantes fue altamente significativa.

De todo esto se concluye que el Plan Bolonia es un modelo prometedor para la mejora de la enseñanza y prevención de la deserción, pero que no está de momento surtiendo los efectos deseados, a pesar de que las evaluaciones externas de seguimiento de títulos implantados han mostrado amplias mejoras (ANECA, 2012).

Las acciones de mejora derivadas de estas conclusiones parecen innumerables y difíciles de alcanzar, sobre todo si se considera el agravante que supone el entorno económico actual, cuyas dificultades han provocado el estancamiento de la plantilla y la imposibilidad de atender con garantías de calidad el volumen de alumnado presente en las aulas, incrementado por aquellos estudiantes que, al no encontrar empleo, han decidido volver a estudiar. Sin embargo, estos hallazgos 
encierran en sí mismos un avance puesto que marcan las pautas a seguir. En este sentido y, a grandes rasgos, proponemos los siguientes:

1) Programas psicoeducativos extracurriculares, que ayuden al alumnado a ser más estratégico, a afrontar y superar los obstáculos, a obtener más éxitos y a ser resilientes.

2) Modalidades docentes basadas en pequeños grupos y con atención más personalizada, donde el profesorado detecte los casos de riesgo y dificultades de aprendizaje, ofrezca feed-back sobre los trabajos realizados, desarrolle metodologías que obliguen al alumnado al trabajo continuado y a esforzarse más y fomente la motivación por los estudios.

3) Modelos de enseñanza alternativos para los estudiantes que prolongan y simultanean estudios y trabajo. A partir de los resultados de las universidades españolas sobre "tasas de eficiencia" (MEC, 2014), en los que se indican que los graduados españoles se matriculan de 53,7 créditos medios, de los cuales se presentan a 45,8 y aprueban 38,7 , se desprende que, o bien la carga de trabajo que se requiere al alumnado no está ajustadas al diseño de los títulos (60 créditos por curso), o que estamos ante estudiantes acomodados y que cada vez trabajan menos. La medida reciente que han tomado las universidades con la "normativa de permanencia y progreso", con la finalidad de disminuir el tiempo que los estudiantes dedican a finalizar la titulación, y la tasa de abandono, nos indicará en el futuro si se trata de una cosa u otra.

En relación con los estudiantes que trabajan y que por su edad tienen otras cargas familiares que les impiden dedicar el tiempo necesario a las clases presenciales, las universidades deberían adaptarse y proponer otros modelos de estudios con estructuras más flexibles, además de la enseñanza on-line que se promueve últimamente. Diferentes estudios han mostrado las dificultades que tienen estos estudiantes por la estructura rígida universitaria (Daza y Elías, 2013; Elías, 2008) y la necesidad de flexibilizar horarios, ofertar actividades alternativas, clases semipresenciales, etc. En una de las encuestas del Eurobarómetro se le pidió a los estudiantes que valoraran distintas propuestas de enseñanza universitaria, y la que más apoyo obtuvo (88\%) fue la de programas a tiempo parcial. Es cierto que el porcentaje de estudiantes que en la unión europea simultanea estudios y trabajo es mayor que en España, pero en un estudio de Sacristán y col (2014) en la universidad de Sevilla, en el que analizaban las causas del absentismo en la universidad, los estudiantes dicen que pueden prepararse la materia con los materiales ofrecidos por el profesor, y que "la relación esfuerzo-resultado" no se corresponde entre sí. 
Efectos del proceso de Bolonia en la reducción del abandono de estudios universitarios: datos para la reflexión y propuestas de mejora

Se estima que en los próximos años el $51 \%$ de los adultos que completará estudios universitarios ingresará en la universidad pasada la edad de 25 años. Esa ya es una realidad en algunos países como Islandia, Israel y Portugal, en los que 1 de cada 3 estudiantes que ingresa en la universidad es mayor de 25 años. El proceso de Bolonia ha dificultado todavía más la combinación de estudios y trabajo, pues el profesorado concluye que es una metodología propia solo para estudiantes presenciales, en lo que también coinciden Daza y Elías (2013).

4) Acciones específicas con estudiantes de riesgo. Las claras intenciones de abandonar sus estudios por parte del $23 \%$ del alumnado encuestado que se desprenden en este trabajo sugieren una intervención urgente sobre ellos. Pero, ¿̇cómo identificar a esos estudiantes de riesgo? Afortunadamente tenemos varios indicadores que se podrían utilizar. Además de estar pendientes de sus expedientes académicos, factor determinante en el abandono, sabemos que los estudiantes que abandonan entran con notas más bajas, no eligen carrera en primera opción, no llevan las tareas al día y no asisten regularmente a clase.

\section{REFERENCIAS BIBLIOGRÁFICAS}

ANECA (2013). Informe sobre el estado de la evaluación externa de la calidad en las universidades españolas. ANECA, 2012. http://www.aneca.es/Sala-de-prensa/Noticias/2013/Estado-de-laevaluacion-de-la-calidad-de-las-universidades-espanolas

ARRIAGA, V., BURILLO, A., CARPEÑO, A. Y CASARAVILLA, A. (2011). Caracterización de los tipos de abandono. Dividamos el problema y venceremos más fácilmente. I Conferencia latinoamericana sobre el abandono en la enseñanza superior (I-CLABES). http://www.alfaguia.org/www-alfa/images/ponencias/clabesl/ ST_1_Abandono/7_JArriaga_Perfiles\%20abandono.pdf

BERNARD, B. (1996). Fostering resilience in urban schools. En Bellinda Williams (ED.), Closing the achievement gaps. Pp. 99-205. Alexandria, Virginia, EE.UU: Association for Supervision and Curriculum Development.

BETHENCOURT, J.T., CABRERA, L., HERNÁNDEZ, J.A., ÁlVAREZ, P. Y GONZÁLEZ, M. (2008). Variables psicológicas y educativas en el abandono universitario. Revista electrónica de investigación psicoeducativa, 6(3), 603-622. 
CABRERA, L., BETHENCOURT, J.T., ALVAREZ, P. Y GONZÁLEZ, M. (2006). El problema del abandono de los estudios universitarios. Revista Electrónica de Investigación y Evaluación Educativa. RELIEVE, 12, 2.

CHRISTIE, H., MUNRO, M., Y FISHER, T. (2004). Leaving university early: exploring the differences between continuing and non-continuing students. Studies in Higher education, 29(5), 617-636.

DOI: http://dx.doi.org/10.1080/0307507042000261580

DAZA, L. Y ELÍAS, M. (2013). ¿Qué quedó de la implantación del espacio europeo de educación superior? Efectos de la crisis en la universidad y panorama actual. Témpora, 15, 1-32.

ELÍAS ANDREU, M. (2008). Los abandonos universitarios: retos ante el Espacio Europeo de Educación Superior. Estudios sobre Educación, $15,101-121$.

GARCÍA DE FANELLI, A.M. (2014). Rendimiento académico y abandono universitario: modelos, resultados y alcance de la producción en la Argentina. Revista argentina de Educación Superior RAES, 6(8), 9-38.

GONZÁLEZ, M., ALVAREZ, P., CABRERA, L., Y BETHENCOURT, J.T. (2007). El abandono de los estudios universitarios: factores determinantes y medidas preventivas. Revista Española de Pedagogía, LXV(236), 71 86.

ISHITANI, T.T. Y DESJARDINS, S.L. (2003). A longitudinal investigation of dropout from colege in the United States. Journal of College Student Retention: Research, Theory and Practice, 4(2), 173-201.

DOI: http://dx.doi.org/10.2190/V4EN-NW42-742Q-2NTL

LANDRY, C.C. (2003). Self-efficay, motivation and outcome expectation correlates of college students' intention certainty. Dissertation Abstracts International Section A. Humanities and Social Sciencies, 64, 3-A, pp.825.

LATIESA, M. (1992). La deserción universitaria. Madrid: CIS.

MARTÍN CABRERA, E. Y GONZÁLEZ AFONSO, M. (Coord.) (2010). El rendimiento académico del alumnado de nuevo ingreso en la Universidad de La Laguna. Servicio de Publicaciones Universidad de La Laguna.

MEC (2014). Datos básicos del sistema universitario español. Curso 2013/14. Ministerio de Educación, Cultura y Deportes. http://www.mecd.gob.es/educacion-mecd/areaseducacion/universidades/estadisticas-informes/datos-cifras.html

MONCADA MORA, L. (2014). La integración académica de los estudiantes universitarios como factor determinante del abandono de corto plazo. Un análisis en el sistema de educación superior a distancia del Ecuador. RIED, 17(2), 173-196.

DOI: http://dx.doi.org/10.5944/ried.17.2.12683

MORALES GIL, F., PARDO ROJAS, A. Y ÁLVAREZ CARPIO, B. (2010). El proceso de convergencia europea en la universidad española, un proyecto en la encrucijada. Revista de Educación, 12, 15-27. 
Efectos del proceso de Bolonia en la reducción del abandono de estudios universitarios: datos para la reflexión y propuestas de mejora

OECD (2010). How many students drop out of tertiary education? in Highlights from Education at a Glance 2010, OECD Publishing. DOI: http://dx.doi.org/10.1787/eag highlights-2010-en

OECD (2014). Education at a glance 2014: OECD indicators, OECD Publishing. DOI: http:/dx.doi.org/10.1787/eag-2014-en

PASCARELLA, E.T. Y TERENZINI, P.T. (1991). How college affects students: findings and insights from twenty years of research. San Francisco, C.A: Jossey Bass.

PETERSON, S.L. (2002). Effects of career decision-making, self-efficacy and degree utility on student persistence: A path analytic study. Journal of college student retention: Research, Theory and Practice, 3(3), 285-299.

REQUES, P. (2007). Atlas digital de la España Universitaria: bases para la planificación estratégica de la enseñanza superior. Santander: Universidad de Cantabria.

SACRISTÁN DÍAZ, M.; GARRIDO VEGA, P.; GONZÁLEZ ZAMORA, M.M.; ALFALLA-LUQUE, R. (2014). ¿2Por qué los alumnos no asisten a clase y no se presentan a los exámenes? Datos y reflexiones sobre absentismo y abandono universitario. Working paper on operationsmanagement.

DOI: http://dx.doi.org/10.4995/wpom.v3i2.1103

SCHNEPF, S. (2014). Do tertiary dropout students really not succeed in european labour markets? Institute for the study of labor (IZA), Bonn, Germany. http://ftp.iza.org/dp8015.pdf

SOLER, I. (2014). Los estudiantes universitarios. Perfiles y modalidades de vinculación con el estudio en la universidad española. Universitat de València.

TADEO LOZANO, J. (2010). Informe de deserción estudiantil. Universidad de Bogotá. Bogotá.

TAYLOR, J.A. Y BEDFORD, T. (2004). Staff perceptions of factors related to non-completion in higher education. Studies in Higher Education, $29(3), 375-394$.

DOI: http://dx.doi.org/10.1080/03075070410001682637

TINTO, V. (1993). Leaving college: Rethinking the causes and cures of student attrition. Chicago: University of Chicago Press.

WANG, M., HAERTEL, G. Y WALBERG, H. (1999). Psychological and educational resilience. En A. Reynolds, H. Walberg y R. Weisburg (Eds.), Promoting positive outcomes. (pp. 99-356). Washington, DC, EE.UU: CWLA Press.

YORKE, M. (2000). The quality of the student experience: what can institutions learn from data relating to non-completion? Quality in Higher Education, 6(1), 61-75.

DOI: http://dx.doi.org/10.1080/13538320050001072 


\section{Sobre la autora:}

\section{Lidia Cabrera Pérez}

Profesora titular del área de Métodos de Investigación y Diagnóstico en Educación en la Universidad de La Laguna. También parte del Programa de "Doctorado en Educación" en la misma universidad, y es miembro de su comisión académica. Su investigación se ha desarrollado entorno a dos líneas, "abandono y rendimiento académico" y "educación intercultural". Sus publicaciones giran alrededor de esos tópico. Ha participado en diferentes proyectos con otras universidades nacionales e internacionales tales como Universidad de Granada, Universidad de Amsterdam, Instituto Politécnico de Costa Rica, Universidad de Massachusset-Boston, etc.

\section{Para citar este artículo:}

Cabrera, L. (2015). Efectos del proceso de Bolonia en la reducción del abandono de estudios universitarios: datos para la reflexión y propuestas de mejora. Revista Fuentes, 16, Junio, pp. 39-62. [Fecha de consulta: dd/mm/aaaa]. http://www.revistafuentes.es/

D.O.I.: http://dx.doi.org/10.12795/revistafuentes.2015.i16.02 
Efectos del proceso de Bolonia en la reducción del abandono de estudios universitarios: datos para la reflexión y propuestas de mejora 